\title{
Paclitaxel and leucovorin-modulated infusional 5-fluorouracil combination chemotherapy for metastatic gastric cancer
}

\author{
BYOUNG CHUL CHO ${ }^{1,3}$, JOO HANG KIM ${ }^{1,3}$, CHOONG BAE KIM ${ }^{4}$, JU HYUK SOHN ${ }^{1,3}$, \\ HYE JIN CHOI ${ }^{1,3}$, YONG CHAN LEE ${ }^{2,3}$ and JOONG BAE AHN ${ }^{1,3}$ \\ ${ }^{1}$ Yonsei Cancer Center, ${ }^{2}$ Division of Gastroenterology, ${ }^{3}$ Department of Internal Medicine and \\ ${ }^{4}$ Department of Surgery, Yonsei University College of Medicine, Seoul, Korea
}

Received August 8, 2005; Accepted October 11, 2005

\begin{abstract}
As no standard chemotherapy regimen has been established for advanced gastric cancer, this study sought to evaluate the efficacy and safety of combination chemotherapy that included paclitaxel and leucovorin (LV)-modulated infusional 5-fluorouracil (5-FU) in metastatic gastric cancer. Patients received a three-hour infusion of $175 \mathrm{mg} / \mathrm{m}^{2}$ of paclitaxel on day 1 . A bolus of $20 \mathrm{mg} / \mathrm{m}^{2}$ of $\mathrm{LV}$ was then administered, followed by a $24-\mathrm{h}$ infusion of $1,000 \mathrm{mg} / \mathrm{m}^{2}$ of 5-FU on days 1 through 3 . The treatment cycle was repeated every 3 weeks until disease progression. Response evaluation was performed according to the RECIST criteria, with toxicity determined by NCI-CTC (version 2.0). A total of 66 patients, including $21(31.8 \%)$ with a history of prior chemotherapy, were enrolled. Fifteen $(71.4 \%)$ of the 21 patients with prior chemotherapy received prolonged infusional 5 -FU. In the 56 evaluable patients ( 37 in the chemotherapynaïve group and 19 in the prior chemotherapy group), tumor responses according to prior exposure to chemotherapy were as follows: 17 (45.9\%) partial response (PR), 6 (16.2\%) stable disease (SD) and $14(37.8 \%)$ progressive disease (PD) in the chemotherapy-naïve group; $1(7.1 \%)$ complete response, 3 (15.8\%) PRs, 8 (42.1\%) SDs and 7 (36.8\%) PDs in the prior chemotherapy group. The overall median response duration was 20 weeks (range, 8-61 weeks), with a median progressionfree survival of 20 weeks [95\% confidence interval (CI), 13.4-26.6 weeks] and 12 weeks (95\% CI, 5.7-18.3 weeks) in the chemotherapy-naïve and prior chemotherapy groups, respectively. The median overall survival was 48 weeks (95\% CI, 38-58 weeks) in the chemotherapy-naïve group and 28 weeks (95\% CI, 22-34 weeks) in the prior chemotherapy group. The most frequent grade III/IV toxicity was neutro-
\end{abstract}

Correspondence to: Dr Joong Bae Ahn, Yonsei Cancer Center, Yonsei University College of Medicine, 134 Shinchon-Dong, Seodaemun-Gu, Seoul 120-752, Korea

E-mail: vvswm513@yumc.yonsei.ac.kr

Key words: palliative chemotherapy, 5-fluorouracil, paclitaxel, gastric cancer penia. Non-hematological toxicity of grade III/IV was rare. Paclitaxel in combination with 5-FU/LV is clinically beneficial for patients with advanced gastric cancer and is a feasible salvage regimen for 5-FU-refractory gastric cancer patients.

\section{Introduction}

Despite its declining incidence in the Western world, gastric cancer is still among the most common malignancies. The control of metastatic gastric cancer has not progressed with medical advances; it remains an incurable disease with a median survival time of only 4-8 months (1). Randomized studies have demonstrated both a survival benefit and a positive impact on quality of life for patients with metastatic gastric cancer when treated with chemotherapy plus supportive care rather than the best supportive care alone $(2,3)$. While it may not cure the disease, chemotherapy has become widely accepted for advanced gastric cancer.

Second-generation regimens for treating advanced gastric cancer are primarily based on 5-fluorouracil (5-FU), high-dose methotrexate, cisplatin, and anthracycline $(1,4-6)$. In phase II trials, response rates of up to $60 \%$ have been reported for regimens such as FAMTX (5-FU, doxorubicin, methotrexate), EAP (etoposide, doxorubicin and cisplatin), ELF (etoposide, leukovorin, 5-FU), FUP (infusional 5-FU, cisplatin), and ECF (epirubicin, cisplatin, infusional 5-FU). In subsequent phase III trials, however, this high level of activity has only been confirmed for the ECF regimen, whereas for the FAMTX, ELF or FUP regimen, response rates were between 20 and $25 \%$ (6-8). In addition, the FAMTX and EAP regimens were associated with severe toxicity. While ECF appears, to date, to be the most active regimen, a definitive standard regimen for the palliative treatment of metastatic gastric cancer has not yet been defined. The need is clear for a new combination regimen so that response rate and survival can be improved in patients with metastatic gastric cancer.

Paclitaxel is one of the most promising cytotoxic agents, acting as a mitotic spindle poison and thereby inducing a mitotic block (9). Moreover, the drug exhibits anti-tumor activity against various tumors, including gastric cancer cell lines (10). As a single agent, it was reported to have overall response rates of between 17 and 29\% (11-13). Results of paclitaxel-containing combinations in the management of gastric cancer are also encouraging (14-19). A three-drug 
combination, including paclitaxel, 5-FU, cisplatin and etoposide, yielded response rates as high as $50 \%$ with a median survival of 7-14 months (14-17). The two-drug combination of paclitaxel and 5-FU, which would expose the patient to fewer harsh side effects, has not been extensively explored as a treatment for gastric cancer, although there have been a few preliminary studies. Murad et al (18) documented a response rate of $65.5 \%$ in 29 patients treated with $5-\mathrm{FU} 1,500 \mathrm{mg} / \mathrm{m}^{2}$ and paclitaxel $175 \mathrm{mg} / \mathrm{m}^{2}$ in a 3 -h infusion every 3 weeks. Bokemeyer et al (19) treated 22 chemonaïve gastric cancer patients with 5-FU as a weekly 24-h continuous infusion plus folinic acid and paclitaxel at 3-week intervals, resulting in a response rate of $32 \%$ and overall survival of 11 months.

The present study sought to evaluate the anti-tumor activity of paclitaxel and LV-modulated infusional 5-FU combination chemotherapy in patients with metastatic gastric cancer. Leucovorin was incorporated into the regimen because most patients with a history of prior chemotherapy had received 5-FU at our institution, and because the addition of LV to 5-FU has been shown to exhibit anti-tumor activity in patients who previously progressed on 5-FU-containing combinations (20).

\section{Materials and methods}

Patient eligibility. Eligibility criteria for this study were as follows: i) histologically-proven adenocarcinoma with metastatic disease; ii) Eastern Cooperative Oncology Group (ECOG) performance scale score 0-2; iii) chemotherapeuticallynaïve or one prior chemotherapy regimen completed at least 3 weeks before entry into study; iv) adjuvant chemotherapy completed at least 6 months before study entry for patients with recurrent disease; v) age $\leq 75$ years; vi) adequate organ function (neutrophil count $\geq 4,000 / \mu 1$, platelet count $\geq 100,000 / \mu 1$, serum total bilirubin $\leq 2 \mathrm{mg} / \mathrm{dl}$, transaminase $\leq 2.5 \mathrm{x}$ normal upper limit, serum creatinine level $\leq 1.5 \mathrm{mg} / \mathrm{dl}$ ); vii) no concurrent uncontrolled medical illnesses or active malignancies. Patients who had undergone palliative resection, and therefore had no measurable lesion on CT scan, were also included in this study. Patients were excluded from the study if they had peripheral neuropathy of grade $\geq 2$ according to the National Cancer Institute's common toxicity criteria (NCI-CTC).

Throughout this report, the term 'palliative resection' refers to the surgical resection of a primary tumor (D2 type resection) with residual tumors remaining grossly in neighboring organs, lymph nodes or peritoneum, or remaining microscopically in the resection margin.

Chemotherapy regimen. Chemotherapy was given according to the following schedule: dexamethasone $20 \mathrm{mg}$, diphenhydramine $25 \mathrm{mg}$ and cimetidine $300 \mathrm{mg}$ administered intravenously (i.v.) $30 \mathrm{~min}$ before paclitaxel for hypersensitivity prophylaxis. A three-hour infusion of $175 \mathrm{mg} / \mathrm{m}^{2}$ of paclitaxel was administered on day 1 . A bolus of $20 \mathrm{mg} / \mathrm{m}^{2}$ of $\mathrm{LV}$, followed by a $24-\mathrm{h}$ infusion of $1,000 \mathrm{mg} / \mathrm{m}^{2}$ of $5-\mathrm{FU}$, was administered once a day for three consecutive days (days 1-3). This chemotherapy course was repeated every three weeks, except in cases of disease progression or patient refusal, for a maximum of 9 cycles. Patients were required to have an absolute neutrophil count (ANC) $\geq 1,500 / \mu 1$ without evidence of active infection, platelet count $\geq 100,000 / \mu 1$, and resolution of any non-hematological toxicity to less than grade 2 before receiving subsequent cycles of chemotherapy. Planned dose modifications included $20 \%$ dose reduction of paclitaxel in cases of grade 3 peripheral neurotoxicity; complete stoppage of paclitaxel in cases of grade 4 skin toxicity or grade 3 anaphylactic reaction; and $20 \%$ dose reduction of paclitaxel and 5-FU in cases of febrile neutropenia, grade IV hematological toxicity, or grade III/IV non-hematological toxicity. Anti-emetic therapy was routinely given prior to chemotherapy. Granulocyte colony stimulating factor (G-CSF) was not planned as a prophylactic aim.

Patient evaluation. For each patient, baseline evaluations included a complete history, physical examination, complete blood count (CBC), serum chemistry and electrolytes, tumor markers (CEA and CA 19-9) and electrocardiogram. Computed tomography (CT) scans of the measurable lesions were performed no more than four weeks prior to treatment. During the course of chemotherapy, CBC was evaluated once a week, with the frequency increasing during the myelosuppressed resting period. Physical examination and performance status, tumor markers and serum chemistry were repeated before each chemotherapy cycle. Tumor evaluation was conducted every two cycles by X-ray, bone scintigraphy and/or CT scan. Toxicity was graded every cycle and calculated as worst toxicity per patient according to NCI-CTC (version 2.0).

The primary efficacy end-point of this study was objective response rate, evaluated according to WHO criteria. Complete response $(\mathrm{CR})$ was defined as the complete disappearance of all measurable disease for a duration of at least four weeks, with partial response (PR) as a $>50 \%$ reduction of all measurable tumor sites. Stable disease (SD) was defined as a $<50 \%$ reduction of tumor lesions and $\leq 25 \%$ progression in tumor diameter. Secondary efficacy end-points included the duration of response, progression-free survival (PFS) and overall survival (OS).

Statistical analysis. PFS defined the period from the start of chemotherapy to the progression of cancer, while OS was from the start of chemotherapy to the date of death or last follow-up. Deaths from all causes were considered in the analysis of overall survival. Response duration was measured from the day of response documentation to the day of disease progression (or death if the patient died without disease progression).

Survival curves were estimated using the Kaplan-Meier method and the differences in survival between the groups were assessed by a log-rank test. Univariate and multivariate analyses were performed using the Cox regression analysis model to identify prognostic factors and the risks associated with them.

\section{Results}

Patient characteristics. Patient characteristics are shown in Table I. A total of 66 patients with a median age of 50 years (range, 24-70 years) were treated in this study. Fifty-five $(83.3 \%)$ patients had an ECOG performance status of 1. Histologically, 17 patients $(25.7 \%)$ had well- to moderately 
Table I. Patients' characteristics $(n=66)$.

\begin{tabular}{lrr}
\hline Characteristic & No. of patients $(\%)$ \\
\hline Sex & & \\
$\quad$ Male & 42 & $(63.6)$ \\
$\quad$ Female & 24 & $(36.4)$ \\
Age (year) & & \\
$\quad$ Median (range) & 50 & $(24-70)$ \\
ECOG & & \\
1 & 55 & $(83.3)$ \\
2 & 11 & $(16.7)$ \\
Histology & & \\
$\quad$ Well/moderately differentiated & 17 & $(25.7)$ \\
Poorly differentiated & 36 & $(54.5)$ \\
Signet ring cell & 13 & $(19.8)$ \\
Stage at diagnosis & & \\
II & & \\
III & 4 & $(6.1)$ \\
IV & 8 & $(12.1)$ \\
\end{tabular}

Previous operation

Unresectable 37

Radical 19

Palliative

No. of disease sites per patient

0

1

2

3

$\geq 4$

$9 \quad(13.6)^{\mathrm{a}}$

$34 \quad(51.5)$

$13 \quad(19.7)$

$8 \quad(12.1)$

$2(3.1)$

Disease sites $^{\mathrm{b}}$

Lymph nodes $\quad 35$

Peritoneum 31

Liver 14

Lung $\quad 4$

Bone 3

Ovary 2

Adrenal

Previous chemotherapy

$\begin{array}{lrl}\text { None } & 45 & (68.2) \\ \text { FAM } & 7 & (10.6) \\ \text { FP } & 8 & (12.1) \\ \text { IP } & 6 & (9.1) \\ \text { Median cycle of previous chemo- } & 6 & (2-12)\end{array}$

therapy (range)

Median RDI of previous chemo-

therapy (range)

$0.86(0.75-0.95)$

aPatients who had undergone palliative resection had no evaluable lesions. ${ }^{\mathrm{b} S o m e}$ patients had more than one site of metastases. ECOG, Eastern Cooperative Oncology Group; FAM, 5-FU + doxorubicin + mitomycin-C; FP, 5-FU + cisplatin; IP, irinotecan + cisplatin differentiated adenocarcinoma, $36(54.5 \%)$ had poorly differentiated adenocarcinoma and 13 (19.8\%) had signet ring cell carcinoma. Nineteen patients $(28.8 \%)$ experienced recurrence after radical gastrectomy. Ten patients had undergone palliative resection immediately prior to the treatment associated with this study. Of these 10 patients, 9 without any measurable lesion on CT scan were excluded from the response assessment but included in the toxicity assessment and survival analysis.

Twenty-three patients $(34.9 \%)$ had multiple metastatic lesions, with the main metastatic sites being the lymph nodes $(n=35)$, peritoneum $(n=31)$ and liver $(n=14)$. The most common non-measurable lesion was bone metastasis $(n=3)$ documented on bone scan. Twenty-one patients $(31.8 \%)$ received chemotherapy that was completed three or more weeks prior to entry into this study. Fifteen $(71.4 \%)$ of the 21 prior chemotherapy patients received prolonged infusional 5-FU, whereas $6(28.6 \%)$ received platinum-based therapy. The median number of cycles of previous chemotherapy was 6 (range, 2-12 cycles), with a median relative dose intensity (RDI) of 0.86 .

Treatment and dose intensity. A total of 338 cycles of chemotherapy were administered, with a median of 6 cycles per patient (range, 2-9 cycles). For chemotherapy naïve patients, the median number of chemotherapy cycles was 6 (range, 2-9 cycles) while, for patients with a history of prior chemotherapy, the median was 4 cycles (range, 2-9 cycles). The median duration of chemotherapy for all patients was 15 weeks (range, 6-27 weeks). Twenty-eight patients (42.4\%) received $\leq 4$ cycles of chemotherapy, with only two of these patients $(7.1 \%)$ expressing the desire to terminate the treatment before the end of the specified cycle. The remaining 38 patients $(57.6 \%)$ received $\geq 5$ cycles, approximately half of whom received $>7$ cycles.

Median actual dose intensities of 5-FU and paclitaxel were $928.1 \mathrm{mg} / \mathrm{m}^{2} /$ week (range, 584.2-1,000 $\mathrm{mg} / \mathrm{m}^{2} /$ week) and $54.9 \mathrm{mg} / \mathrm{m}^{2} /$ week (range, 26.3-58.0 mg/m²/week), respectively. The median relative dose intensities (RDI) of 5-FU and paclitaxel were 0.93 (range, 0.58-1.0) and 0.94 (0.58-1.0), respectively.

There were 30 cases $(45.5 \%)$ of chemotherapy delay or dose reduction. The causes of dose reduction or schedule delay were as follows: $13(43.4 \%)$ neutropenia, $9(30 \%)$ requests from patients, 4 (13.3\%) febrile neutropenia, $3(10 \%)$ non-hematological toxicity and $1(3.3 \%)$ sepsis due to urinary tract infection.

Efficacy. A total of 56 patients were assessable for response (37 in the chemotherapy-naïve group and 19 in the prior chemotherapy group). The remaining 10 patients were not assessable for response either because they had no measurable lesion (9 patients, 7 in the chemotherapy-naïve group and 2 in the prior chemotherapy group) or they refused (1 patient in the chemotherapy-naïve group). The objective response rates (ORRs) according to prior chemotherapy are listed in Table II.

Among 37 chemotherapy-naïve patients, 17 showed a PR. The ORR was $45.9 \%$. Six patients had SD, so the resulting disease control rate (DCR) reached $62.2 \%$. The median time to response in this group was 8 weeks, while the median response duration was 20 weeks (range, 8-61 weeks). 
Table II. Response evaluation $(\mathrm{n}=56)^{\mathrm{a}}$.

\begin{tabular}{lcccccc}
\hline & \multicolumn{3}{c}{ Response (n) } & & \\
\cline { 2 - 5 } Treatment group & CR & PR & SD & $\begin{array}{c}\text { ORR } \\
(\%)\end{array}$ & $\begin{array}{c}\text { DCR } \\
(\%)\end{array}$ \\
\hline Chemotherapy-naïve $(\mathrm{n}=37)$ & 0 & 17 & 6 & 45.9 & 62.2 \\
Prior chemotherapy $(\mathrm{n}=19)$ & 1 & 3 & 8 & 21.1 & 63.2 \\
\hline
\end{tabular}

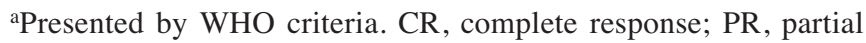
response; $\mathrm{SD}$, stable disease; ORR, objective response rate; DCR, disease control rate $(\mathrm{CR}+\mathrm{PR}+\mathrm{SD})$.

Table III. Response evaluation by metastasis sites.

\begin{tabular}{lcccccc}
\hline $\begin{array}{l}\text { Metastatic } \\
\text { sites }\end{array}$ & $\begin{array}{c}\text { No.of evaluable } \\
\text { lesions }\end{array}$ & CR & PR & SD & PD & RR $^{\mathrm{a}}$ \\
\hline Lymph nodes & 35 & 0 & 15 & 7 & 13 & 42.9 \\
Peritoneum & 31 & 1 & 9 & 11 & 10 & 32.3 \\
Liver & 14 & 0 & 4 & 3 & 7 & 28.6 \\
Lung & 4 & 0 & 1 & 1 & 2 & 25.0 \\
Bone & 3 & 0 & 1 & 0 & 2 & 33.3 \\
Ovary & 2 & 0 & 2 & 0 & 0 & 100 \\
Adrenal & 1 & 0 & 0 & 0 & 1 & 0 \\
\hline
\end{tabular}

$\mathrm{CR}$, complete response; PR, partial response; $\mathrm{SD}$, stable disease; $\mathrm{PD}$,

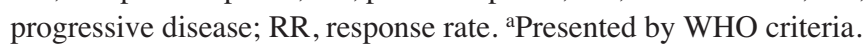

Among 19 patients with a history of chemotherapy, there was $1 \mathrm{CR}$ and $3 \mathrm{PRs}$, resulting in an ORR of $21.1 \%$. Eight patients had SD; the resulting DCR was $63.2 \%$. As for the other group, the median time to response was 8 weeks, although the median response duration was significantly less at 8 weeks (range, 8-12 weeks). One patient with a CR presented with a tumor at the primary site and peritoneal nodules. Following palliative gastrectomy and four cycles of chemotherapy, CR was proven by CT scan. The duration of $\mathrm{CR}$ in the patient is currently $24+$ weeks. When the response rate was analyzed according to metastatic sites, similar results were found (Table III).

Survival. With a median follow-up du ation of 32 weeks (range, 12-76 weeks), 58 patients had progressed, while 8 patients had not. The most common site of progression was carcinomatosis. Of the 58 patients who had progressed, 43 patients $(74.1 \%$, 29 in the chemotherapy-naïve group and 14 in the prior chemotherapy group) were switched to a salvage regimen.

The median PFS was 20 weeks (95\% CI, 13.4-26.6 weeks) for the chemotherapy-naïve group and 12 weeks (95\% CI, 5.7-18.3 weeks) for the prior chemotherapy group (Fig. 1). The median OS for the same groups was 48 weeks (95\% CI, 38-58 weeks) and 28 weeks (95\% CI, 22-34 weeks),

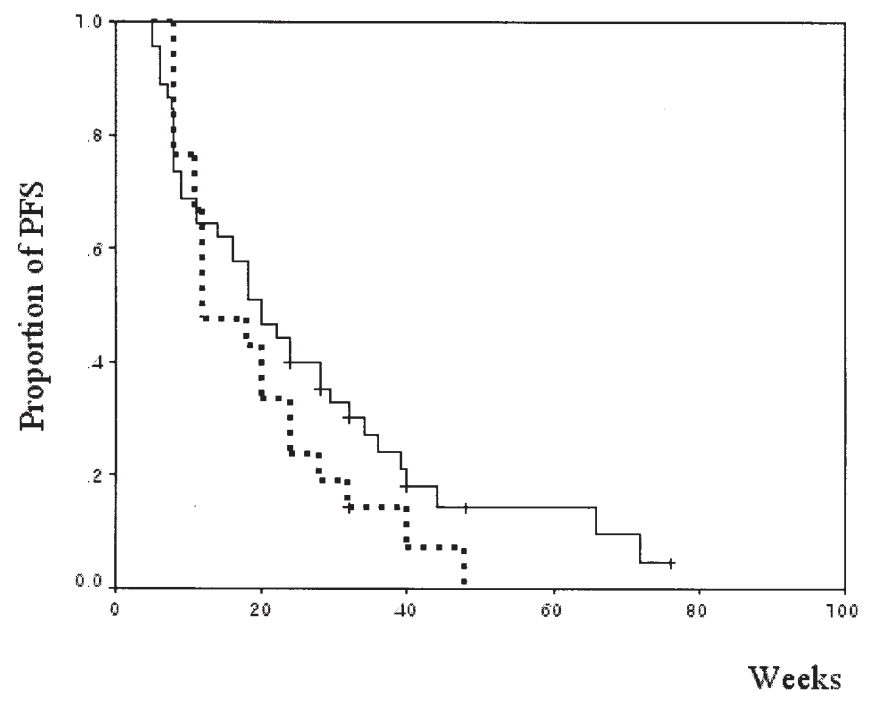

Figure 1. Progression-free survival according to history of prior chemotherapy. The median PFS was 20 weeks for the chemotherapy-naïve group ( - ) and 12 weeks (-----) for the prior chemotherapy group, respectively.

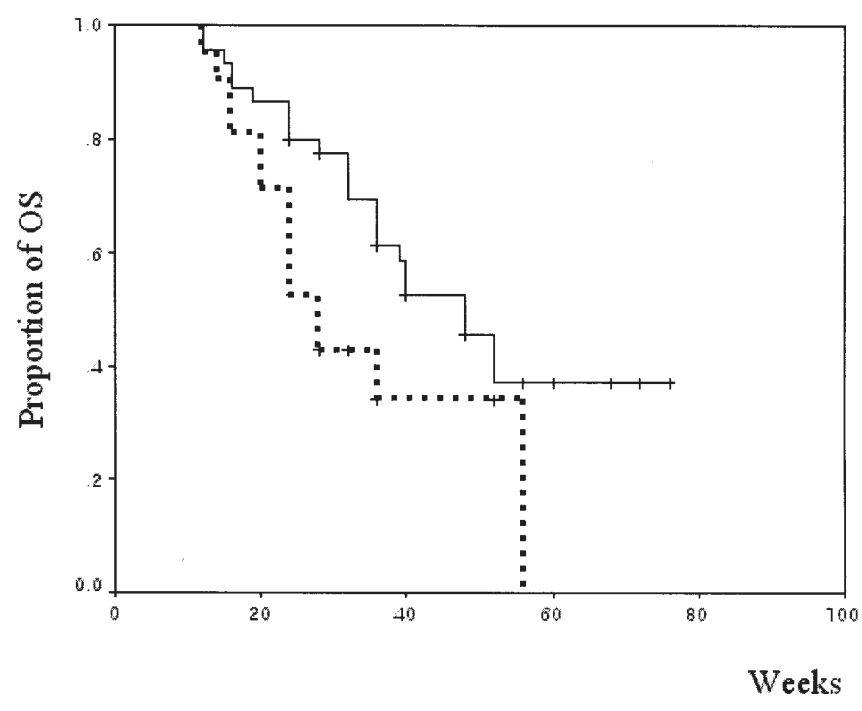

Figure 2. Overall survival according to history of prior chemotherapy. The median OS was 48 weeks for the chemotherapy-naïve group ( - ) and 28 weeks (------) for the prior chemotherapy group, respectively.

respectively (Fig. 2). The 1-year OS rates were $45.7 \%$ for the chemotherapy-naïve group and $34.3 \%$ for the prior chemotherapy group. The median PFS of palliatively-resected patients was significantly higher than that of unresected patients (40 vs. 14 weeks, $\mathrm{P}=0.0007)$. The median $\mathrm{OS}$ was not reached for palliatively-resected patients, and the 1-year OS rate, at $88.9 \%$, was significantly higher than unresected patients $(\mathrm{P}=0.022)$.

Prognostic factors for survival. Univariate and multivariate analyses for the prognostic value of PFS and OS were performed (Table IV). In the univariate analysis, the response to chemotherapy and palliative resection were selected to be significant for PFS, whereas the response to chemotherapy, palliative resection, histology and history of previous chemotherapy were the important variables influencing OS. In 
Table IV. Prognostic factors influencing progression-free survival and overall survival.

\begin{tabular}{llccc}
\hline & \multicolumn{2}{c}{ Progression-free survival } & \multicolumn{2}{c}{ Overall survival } \\
\cline { 2 - 5 } & $\begin{array}{c}\text { Univariate } \\
\text { P-value }\end{array}$ & $\begin{array}{c}\text { Multivariate } \\
\text { P-value }\end{array}$ & $\begin{array}{c}\text { Univariate } \\
\text { P-value }\end{array}$ & $\begin{array}{c}\text { Multivariate } \\
\text { P-value }\end{array}$ \\
\hline Sex & 0.704 & $\mathrm{NS}$ & 0.156 & $\mathrm{NS}$ \\
Age & 0.263 & $\mathrm{NS}$ & 0.055 & $\mathrm{NS}$ \\
ECOG (1 vs. 2) & 0.301 & $\mathrm{NS}$ & 0.099 & $\mathrm{NS}$ \\
Histology & 0.108 & $\mathrm{NS}$ & 0.023 & $\mathrm{NS}$ \\
Previous chemotherapy & 0.407 & $\mathrm{NS}$ & 0.017 & $\mathrm{NS}$ \\
Response to chemotherapy & 0.0048 & 0.002 & 0.0026 & 0.013 \\
Palliative resection & 0.003 & 0.004 & 0.012 & 0.014 \\
\hline
\end{tabular}

NS, not significant.

Table V. Adverse reactions.

\begin{tabular}{|c|c|c|c|c|c|}
\hline \multirow[b]{2}{*}{ Adverse reaction } & \multicolumn{4}{|c|}{ Grade (no. of patients) } & \multirow{2}{*}{$\begin{array}{c}\text { Grade III } \\
\text { and IV }(\%)\end{array}$} \\
\hline & I & II & III & IV & \\
\hline \multicolumn{6}{|l|}{ Hematological } \\
\hline Neutropenia & 5 & 7 & 18 & 13 & 47.0 \\
\hline Anemia & 9 & 29 & 1 & 0 & 1.5 \\
\hline Thrombocytopenia & 17 & 0 & 1 & 0 & 1.5 \\
\hline Febrile neutropenia & 0 & 0 & 4 & 0 & 6.1 \\
\hline \multicolumn{6}{|l|}{ Non-hematological } \\
\hline Nausea/vomiting & 13 & 3 & 0 & 0 & 0 \\
\hline Diarrhea & 12 & 2 & 1 & 0 & 1.5 \\
\hline Infection & 0 & 1 & 1 & 0 & 1.5 \\
\hline Fever & 1 & 0 & 0 & 0 & 0 \\
\hline $\begin{array}{l}\text { Hypersensitivity } \\
\text { reaction }\end{array}$ & 0 & 0 & 0 & 0 & 0 \\
\hline $\begin{array}{l}\text { Peripheral } \\
\text { neuropathy }\end{array}$ & 13 & 11 & 1 & 0 & 1.5 \\
\hline Myalgia & 15 & 0 & 0 & 0 & 0 \\
\hline Mucositis & 26 & 2 & 0 & 0 & 0 \\
\hline Lethargy & 24 & 12 & 0 & 0 & 0 \\
\hline
\end{tabular}

multivariate analysis, the response to chemotherapy and palliative resection showed an independent prognostic effect on both PFS and OS.

Toxicity. All 66 patients in the study were assessed for toxicity. The frequencies of hematological and non-hematological adverse events are shown in Table $\mathrm{V}$. The most common grade III and IV hematological toxicity was neutropenia, found in 27.3 and $19.7 \%$ of patients, respectively. Febrile neutropenia was documented in four patients $(6.1 \%)$ and completely recovered with supportive care. The incidence of grade III/ IV neutropenia was similar in the chemotherapy- naïve and previous chemotherapy patients $(\mathrm{P}=0.253)$. Grade III anemia and thrombocytopenia were documented in $1.5 \%$ of patients in both groups.

Common non-hematological toxicity included lethargy $(54.5 \%)$, mucositis $(42.4 \%)$, and peripheral neuropathy $(37.9 \%)$, although grade III toxicities were observed only as peripheral neuropathy $(1.5 \%)$ and diarrhea (1.5\%). Most patients with peripheral neuropathy required low-dose analgesics. There was one case of grade III infection during chemotherapy (urinary tract infection) that was recovered completely by supportive care. There was no treatment-related mortality.

\section{Discussion}

The present study is one of few reports on the efficacy and safety of the combination treatment of paclitaxel plus LVmodulated 5-FU in patients with metastatic gastric cancer. The rationale for the combined use of these drugs was: a) documented activity of both drugs in gastric cancer when used individually $(1,10-13)$; b) the combination of 5-FU and LV has shown activity in patients who have previously progressed on 5-FU-containing combinations (20 ); c) apparent additive cytotoxicity and safety of this combination in patients with breast and esophageal cancer $(21,22)$; and d) encouraging results of recently published reports of treatment with a combination of 5-FU and the semi-synthetic taxoid, docetaxel (23).

With an ORR of $45.9 \%$, median PFS of 20 weeks, and median OS of 48 weeks for the chemotherapy-naïve group, the results of the present study suggest high anti-tumor activity for this combination of agents in metastatic gastric cancer. The observed anti-tumor potential, in agreement with the previously mentioned phase II studies of Murad et al (ORR 65.5\%, median survival 12 months), and Bokemeyer et al (ORR 32\%, median survival 11 months), indicates that paclitaxel/5-FU-based combination chemotherapy might be as active as second-generation regimens, including the ECF regimen. Our results further suggest that this combination is at least as efficacious as more intense and toxic three-drug 
combinations such as the PFC (paclitaxel, 5-FU and cisplatin) or TPE (paclitaxel, cisplatin, etoposide) regimens (14-17). Consequently, the 5-FU/paclitaxel combination is a possible option for patients with poor performance status or cardiac and renal dysfunction unsuitable for intensive hydration. These promising results must be interpreted with caution, however, until they face confirmation in a randomized trial setting.

Interestingly, the DCRs between the chemotherapy-naïve and prior chemotherapy groups were similar (62.2 and 63.2\%, respectively). One possible explanation for the high DCR, even for patients who had previously been treated with 5-FU, is that the addition of LV to 5-FU has activity in patients who have previously progressed on 5-FU-containing combinations (20). The combination of paclitaxel and 5-FU also has an additive cytotoxicity and different mechanism of action (24).

The median OS and TTP achieved in our study are also comparable with those described in other studies. Of particular note are the results of chemotherapy-naïve patients; the overall survival of 48 weeks was at the higher end of the survival achieved by other combination studies.

In our cohorts, several factors may have either positively or negatively influenced survival. First, one would expect a high tumor burden to hinder patient survival. About $35 \%$ of our patients had multiple metastatic lesions, and $47 \%$ had combined peritoneal seeding. Even with these high tumor burdens, our regimen induced a considerable DCR $(63 \%)$, reaffirming the activity of paclitaxel and 5-FU/LV in advanced gastric cancer. Second, the fact that $71.4 \%$ of the 21 patients with a history of prior chemotherapy received prolonged infusional 5-FU was of great concern. Third, palliative resection, which was an important variable in multivariate analysis, might have exerted a beneficial influence on survival in both chemotherapy-naïve and prior chemotherapy patients $(25,26)$.

When assessing the value of an anticancer treatment, it is important to consider the impact on quality of life, determined principally by the toxicity of the chemotherapy. This is particularly so for patients with advanced gastric cancer, whose life expectancy is short. With this in mind, our combination regimen was generally well-tolerated. In this study and others, 5-FU was administered as a protracted, 24-h infusion, since this mode of action appears to be less toxic $(14-16,19)$. The primary limiting toxicity was neutropenia, although this toxicity can potentially be overcome by prophylactic administration of G-CSF to patients.

Although the hematological toxicity in our study was nearly the same as in other reports, our regimen seems preferable in terms of tolerability of non-hematological toxicity. While peripheral neuropathy, a side effect specific to paclitaxel, occurred in $37.9 \%$ patients, grade III neuropathy occurred in just $1.5 \%$ of patients and was manageable with low-dose analgesics. The incidence of this reaction tended to increase with repeated administration of the drug, and improvements were noted in most patients after the end of chemotherapy. The high RDI of our regimen suggests that the toxicity, especially the non-hematological toxicity, was quite manageable.

In conclusion, paclitaxel in combination with $5-\mathrm{FU} / \mathrm{LV}$ is active in patients with advanced gastric cancer and is a feasible salvage regimen for 5-FU-resistant patients. The results of this study suggest that this regimen is at least as efficacious as second-generation regimens or paclitaxel-containing threedrug combinations.

\section{References}

1. Wohrer SS, Raderer and Hejna M: Palliative chemotherapy for advanced gastric cancer. Ann Oncol 15: 1585-1595, 2004

2. Glimelius B, Ekstrom K, Hoffman K, Graf W, Sjoden PO, Haglund U, Svensson C, Enander LK, Linne T, Sellstrom H and Heuman R: Randomized comparison between chemotherapy plus best supportive care with best supportive care in advanced gastric cancer. Ann Oncol 8: 163-168, 1997.

3. Pyrhonen S, Kuitunen T, Nyandoto P and Kouri M: Randomised comparison of fluorouracil, epidoxorubicin and methotrexate (FEMTX) plus supportive care with supportive care alone in patients with non-resectable gastric cancer. Br J Cancer 71: 587-591, 1995.

4. Wils JA, Klein HO, Wagener DJ, et al: Sequential high-dose methotrexate and fluorouracil combined with doxorubicin-a step ahead in the treatment of advanced gastric cancer: a trial of the European Organization for Research and Treatment of Cancer Gastrointestinal Tract Cooperative Group. J Clin Oncol 9: 827-831, 1991.

5. Preusser P, Wilke H, Achterrath W, Fink U, Lenaz L, Heinicke A, Meyer J, Meyer HJ and Buente H: Phase II study with the combination etoposide, doxorubicin and cisplatin in advanced measurable gastric cancer. J Clin Oncol 7: 1310-1317, 1989.

6. Vanhoefer U, Rougier P, Wilke H, Ducreux MP, Lacave AJ, van Cutsem E, Planker M, Santos JG, Piedbois P, Paillot B, Bodenstein H, Schmoll HJ, Bleiberg H, Nordlinger B, Couvreur ML, Baron B and Wils JA: Final results of a randomized phase III trial of sequential high-dose methotrexate, fluorouracil, and doxorubicin versus etoposide, leucovorin, and fluorouracil versus infusional fluorouracil and cisplatin in advanced gastric cancer: a trial of the European Organization for Research and Treatment of Cancer Gastrointestinal Tract Cancer Cooperative Group. J Clin Oncol 18: 2648-2657, 2000.

7. Kelsen D, Atiq OT, Saltz L, Niedzwiecki D, Ginn D, Chapman D, Heelan R, Lightdale C, Vinciguerra V and Brennan M: FAMTX versus etoposide, doxorubicin, and cisplatin: a random assignment trial in gastric cancer. J Clin Oncol 10: 541-548, 1992.

8. Waters JS, Norman A, Cunningham D, et al: Long-term survival after epirubicin, cisplatin and fluorouracil for gastric cancer: results of a randomized trial. Br J Cancer 80: 269-272, 1999.

9. Rowinsky EK, Cazenave LA and Donehover RC: Taxol: a novel investigational antimicrotubule agent. J Natl Cancer Inst 82: 1247-1259, 1990.

10. Chang YF, Li LL, Wu CW, Liu TY, Lui WY, P'eng FK and Chi CW: Paclitaxel-induced apoptosis in human gastric carcinoma cell lines. Cancer 77: 14-18, 1996.

11. Ajani JA, Fairweather J, Dumas P, Patt YZ, Pazdur R and Mansfield PF: Phase II study of taxol in patients with advanced gastric carcinoma. Cancer J Sci Am 4: 269-274, 1998.

12. Cascinu S, Graziano F, Cardarelli N, Marcellini M, Giordani P, Menichetti ET and Catalano G: Phase II study of paclitaxel in pretreated advanced gastric cancer. Anticancer Drugs 9: 307-310, 1998.

13. Yamaguchi K, Tada M, Horikoshi N, Otani T, Takiuchi H, Saitoh S, Kanamaru R, Kasai Y, Koizumi W, Sakata Y and Taguchi T: Phase II study of paclitaxel with 3-h infusion in patients with advanced gastric cancer. Gastric Cancer 5: 90-95, 2002.

14. Kim YH, Shin SW, Kim BS, Kim JH, Kim JG, Mok YJ, Kim CS, Rhyu HS, Hyun JH and Kim JS: Paclitaxel, 5-fluorouracil and cisplatin combination chemotherapy for the treatment of advanced gastric carcinoma. Cancer 15: 295-301, 1999.

15. Kollmannsberger C, Quietzsch D, Haag C, Lingenfelser T, Schroeder M, Hartmann JT, Baronius W, Hempel V, Clemens M, Kanz L and Bokemeyer C: A phase II study of paclitaxel, weekly, 24-h continous infusion 5-fluorouracil, folinic acid and cisplatin in patients with advanced gastric cancer. Br J Cancer 83: 458-462, 2000.

16. Honecker F, Kollmannsberger C, Quietzsch D, Haag C, Schroeder M, Spott C, Hartmann JT, Baronius W, Hempel V, Kanz L and Bokemeyer C: Phase II study of weekly paclitaxel plus 24-h continuous infusion 5-fluorouracil, folinic acid and 3-weekly cisplatin for the treatment of patients with advanced gastric cancer. Anticancer Drugs 13: 497-503, 2002. 
17. Lokich JJ, Sonneborn H, Anderson NR, Bern MM, Coco FV, Dow E and Oliynyk P: Combined paclitaxel, cisplatin and etoposide for patients with previously untreated esophageal and gastroesophageal carcinomas. Cancer 85: 2347-2351, 1999.

18. Murad AM, Petroianu A, Guimaraes RC, Aragao BC, Cabral LO and Scalabrini-Neto AO: Phase II trial of the combination of paclitaxel and 5-fluorouracil in the treatment of advanced gastric cancer: a novel, safe and effective regimen. Am J Clin Oncol 22: 580-586, 1999.

19. Bokemeyer C, Hartmann JT, Lampe CS, Clemens MR, Quietzsch D, Forkmann L and Kanz L: Paclitaxel and weekly 24-h infusion of 5-fluorouracil/folinic acid in advanced gastric cancer. Semin Oncol 24 (suppl. 19): 96-100, 1997

20. Arbuck SG, Douglass HO Jr, Trave F, Milliron S, Baroni M, Nava H, Emrich LJ and Rustum YM: A phase II trial of 5fluorouracil and high-dose intravenous leucovorin in gastric carcinoma. J Clin Oncol 5: 1150-1156, 1987.

21. Klaassen U, Wilke H and Seeber S: Paclitaxel combined with weekly high-dose 5-fluorouracil/folinic acid and cisplatin in the treatment of advanced breast cancer. Semin Oncol 23: 32-37, 1996.
22. Schnirer II, Komaki R, Yao JC, Swisher S, Putnam J, Pisters PW, Roth JA and Ajani JA: Pilot study of concurrent 5-fluorouracil/ paclitaxel plus radiotherapy in patients with carcinoma of the esophagus and gastroesophageal junction. Am J Clin Oncol 24: 91-95, 2001

23. Constenla M, Garcia-Arroyo R, Lorenzo I, Carrete N, Campos B and Palacios P: Docetaxel, 5-fluorouracil and leucovorin as treatment for advanced gastric cancer: results of a phase II study. Gastric Cancer 5: 142-147, 2002.

24. Kano Y, Akutsu M, Tsunoda S, Ando J, Matsui J, Suzuki K, Ikeda T, Inoue Y and Adachi K: Schedule-dependent interaction between paclitaxel and 5-fluorouracil in human carcinoma cell lines in vitro. Br J Cancer 74: 704-710, 1996.

25. Hallissey MT, Allum WH, Roginski C and Fielding JW: Palliative surgery for gastric cancer. Cancer 62: 440-444, 1988.

26. Doglietto GB, Pacelli F, Caprino P, Alfieri S, Carriero C, Malerba $\mathrm{M}$ and Crucitti F: Palliative surgery for far-advanced gastric cancer: a retrospective study on 305 consecutive patients. Am Surg 65: 352-355, 1999. 